\title{
Significant association between tumor mutational burden and immune-related adverse events during immune checkpoint inhibition therapies
}

\author{
Csaba Kerepesi $^{1,6} \cdot$ Tibor Bakacs $^{2} \cdot$ Ralph W. Moss $^{3} \cdot$ Shimon Slavin $^{4} \cdot$ Colin C. Anderson $^{5}$ (D)
}

Received: 20 November 2019 / Accepted: 21 February 2020 / Published online: 9 March 2020

(c) The Author(s) 2020

\begin{abstract}
More than 2000 immuno-oncology agents are being tested or are in use as a result of the cancer immunotherapy revolution. Manipulation of co-inhibitory receptors has achieved tumor eradication in a minority of patients, but widespread immunerelated adverse events (irAEs) compromised tolerance to healthy self-tissues in the majority. We have proposed that a major mechanism of irAEs is similar to a graft-versus-malignancy effect of graft-versus-host disease. To verify our hypothesis, we retrieved post-marketing data of adverse events from the U.S. Food and Drug Administration Adverse Event Reporting System. A significant positive correlation was revealed in 7677 patients between the reporting odds ratio of irAEs during immune checkpoint inhibitor therapy and the corresponding tumor mutational burden across 19 cancer types. These results can be interpreted to mean that the ICI drugs unleashed T cells against "altered-self," self, and tumors resulting in better overall survival.
\end{abstract}

Keywords Immune-related adverse event · Immune checkpoint inhibition · Tumor mutation burden · Graft-versus-host disease · FAERS

Electronic supplementary material The online version of this article (https://doi.org/10.1007/s00262-020-02543-6) contains supplementary material, which is available to authorized users.

Csaba Kerepesi

kerepesi@sztaki.hu

Colin C. Anderson

colinand@ualberta.ca

1 Institute for Computer Science and Control (SZTAKI), Kende u 13-17, Budapest 1111, Hungary

2 PRET Therapeutics Ltd., Budapest 1124, Hungary

3 Moss Reports, 104 Main Street Unit 1422, Blue Hill, ME 04614-1422, USA

4 Biotherapy International, The Center for Innovative Cancer Immunotherapy and Cellular Medicine, Weizmann Center, 14 Weizmann Street Floor 15, Suite 1503, 64239 Tel Aviv, Israel

5 Departments of Surgery and Medical Microbiology and Immunology, Alberta Diabetes Institute, Alberta Transplant Institute, University of Alberta, Edmonton, AB T6G 2E1, Canada

6 Present Address: Brigham and Women's Hospital, Harvard Medical School, 77 Avenue Louis Pasteur, Boston, MA 02115, USA

\author{
Abbreviations \\ AE Adverse event \\ FAERS Food and Drug Administration Adverse Event \\ Reporting System \\ GVHD Graft-versus-host disease \\ GVM Graft-versus-malignancy \\ ICI Immune checkpoint inhibitor \\ irAE Immune-related adverse event \\ NSCLC Non-small cell lung carcinoma \\ ROR Reporting odds ratio \\ TMB Tumor mutation burden
}

Bomze et al. found a significant association between tumor mutational burden (TMB) and immune-related adverse events (irAEs) across different cancer types during nivolumab or pembrolizumab anti-programmed cell death-1 (anti-PD-1) therapy [1]. This finding seems to support our hypothesis that the widespread irAEs are primarily due to a mechanism similar to autologous-graft-versus-hostlike-disease (auto-GVHD), part of which is a graft-versusmalignancy (GVM) effect responsible for induction of anti-cancer effects that can result in tumor eradication [2]. Since different agents targeting PD-1, PD-L1, or cytotoxic 
T-lymphocyte-associated protein 4 (CTLA-4) may involve distinct mechanisms, we examined whether the correlation of TMB and irAEs with nivolumab or pembrolizumab therapy is applicable to immune checkpoint blockade more generally. Formal proof that irAEs are associated with a significantly longer survival is available for anti-PD-1 (pembrolizumab) [3]. Notwithstanding, the highest efficacy can only be achieved with concurrent ipilimumab and nivolumab blockade, which is also inseparable from the highest rate of irAEs. The record 3-year overall survival (OS) rate of 63\% has been observed in patients with metastatic melanoma who were treated with combined ipilimumab and nivolumab blockade [4]. For this spectacular result, however, a heavy price had to be paid: tolerance to healthy self-tissues was severely compromised. Treatment-related irAEs, of anygrade, were reported in $92.6 \%$ of patients, $40.4 \%$ of which were grade 3 and 4 , leading to discontinuation in $24.5 \%$ of patients and one death. Not unexpectedly, a meta-analysis including 48 trials with 7936 patients who were treated with nivolumab or nivolumab plus ipilimumab raised the question whether the deleterious effects of severe irAEs outweigh the benefit from the addition of ipilimumab [5]. We have therefore also included in our analysis the anti-CTLA-4/anti-PD-1 combination therapy.

We strictly followed Bomze et al. [1] and retrieved post-marketing data of adverse events from the U.S. Food and Drug Administration Adverse Event Reporting System (FAERS) from July 1, 2014, to March 31, 2019. We considered cancers only for which there were at least 100 cases of adverse events during immune checkpoint inhibitor therapy reported in FAERS. To assess the risk of a patient developing any irAE (Supplementary Table S1) as defined by the reporting odds ratios (RORs) [6] we compared the odds of reporting these irAEs in patients treated either with anti-CTLA-4 (ipilimumab, tremelimumab), anti-PD-1 (nivolumab, pembrolizumab, cemiplimab, pidilizumab, spartalizumab, tislelizumab, toripalimab), anti-PD-L1 (avelumab, durvalumab, atezolizumab), or combination therapy with the odds for all other drugs in the database. The median number of coding somatic mutations per megabase in tumor tissue (referred as tumor mutation burden, TMB) for each cancer type was obtained from previously published comprehensive genomic profiling [7, 8]. GVHD symptoms and FAERS search terms were determined according to Jagasia et al. [9] and collected into Supplementary Table S2.

Our search strategy identified a total of 80,193 adverse events (AEs) of all types in 28,092 patients reported as treated with immune checkpoint inhibitor therapy for 19 different cancer types. Of these patients, 7677 had at least 1 irAE (proportion: 27.3\%; proportion per cancer: 9.1-36\%). The comparator group comprised 17,069,184 AE reports from 5,937,270 patients. Of these patients, 428,922 had at least 1 irAE (proportion: 7.2\%; odds: 0.078). Our analysis revealed a significant positive correlation between the ROR of reporting an irAE during immune checkpoint inhibitor therapy and the corresponding TMB across multiple cancer types, with a higher ROR of irAE associated with a higher median number of coding somatic mutations per megabase of DNA (Fig. 1; Pearson correlation coefficient $r=0.68$, $p=0.0012$ ). Importantly, significant positive association was also demonstrated when exclusively all anti-PD-1/antiCTLA-4 combination treatments were included (Fig. 2;
Fig. 1 Association between tumor mutational burden (median number of coding somatic mutations per megabase) and immune-related adverse events during either Anti-CTLA-4, Anti-PD-1, AntiPD-L1 or combination therapy. The straight line represents the linear fit. Circle size and color represent the total number of FAERS cases for each cancer type. Pearson correlation coefficient $(r)$ and the corresponding $p$ value are shown at the bottom-right of the figure. The $p$ value means the probability of getting higher $r$ with random ROR values

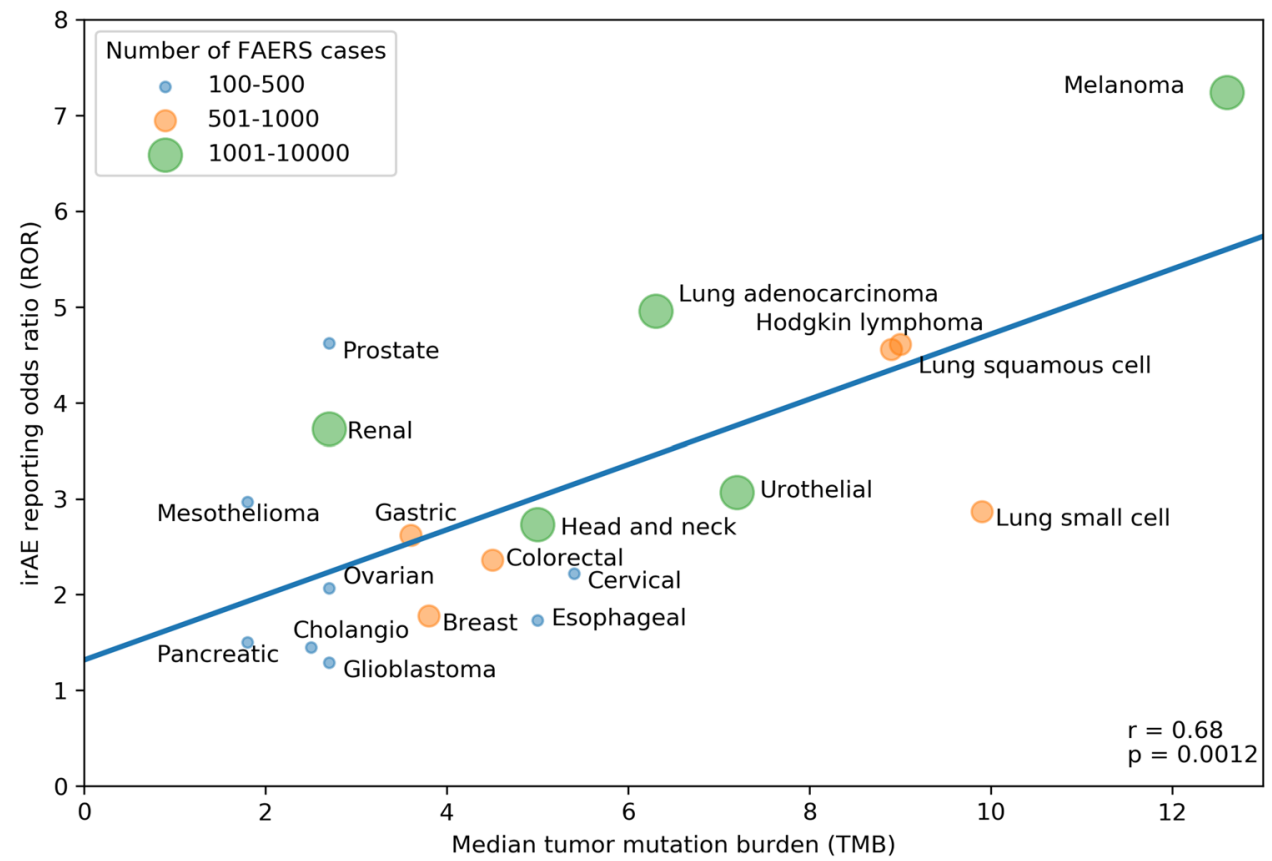


Fig. 2 Association between tumor mutational burden (median number of coding somatic mutations per megabase) and immune-related adverse events during AntiCTLA-4/Anti-PD-1 combination therapy. The straight line represents the linear fit. Circle size and color represent the total number of FAERS cases for each cancer type. Pearson correlation coefficient $(r)$ and the corresponding $p$ value are shown at bottom-right of the figure. The $p$ value means the probability of getting higher $r$ with random ROR values

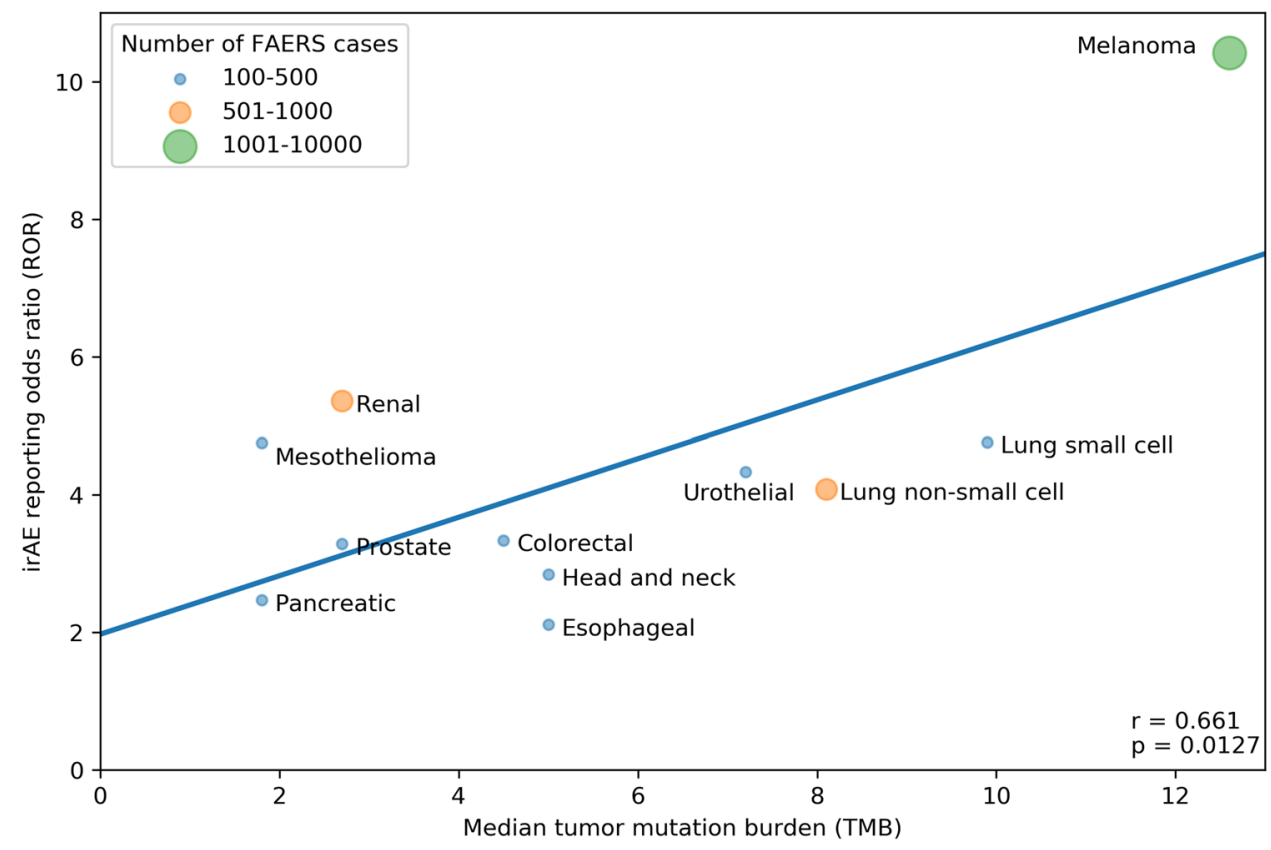

$r=0.661, p=0.0127)$. Since there is an extensive overlap between the irAEs of ICIs [10] and the symptoms of chronic graft-versus-host disease (GVHD) [9], we investigated the correlation between the chronic GVHD-related AE reporting odds ratio (ROR) and the corresponding TMB across multiple cancer types. A significant positive association was demonstrated (Fig. 3; $r=0.466, p=0.0217$ ). This association appears not to be widely recognized. We found 3172 and 4102 papers with the keywords either <ipilimumab $>$ or $<$ nivolumab $>$, surprisingly, only 13 and 17 papers using the keywords either $<$ ipilimumab and GVHD $>$ or $<$ nivolumab and GVHD $>$, respectively (PubMed search as of November 2019). While out of these 30 papers 27 were concerned with the effect of ICI drugs on GVHD after allogeneic hematopoietic stem cell transplantation, only our own papers proposed the possibility of an ICI drug induced auto-GVHD reaction $[2,11,12]$.

High TMB, representing genomic instability, has the potential to induce neoantigen production. Alexandrov et al. [13] analyzed 4,938,362 mutations from 7042 cancers and extracted more than 20 distinct mutational signatures. The prevalence of somatic mutations was found to
Fig. 3 Association between tumor mutational burden (median number of coding somatic mutations per megabase) and chronic graftversus-host disease (cGVHD) related adverse events (AEs) during either Anti-CTLA-4, Anti-PD-1, Anti-PD-L1 or combination therapy. The straight line represents the linear fit. Circle size and color represent the total number of FAERS cases for each cancer type. Pearson correlation coefficient $(r)$ and the corresponding $p$ value are shown at the bottom-right of the figure. The $p$ value means the probability of getting higher $r$ with random ROR values

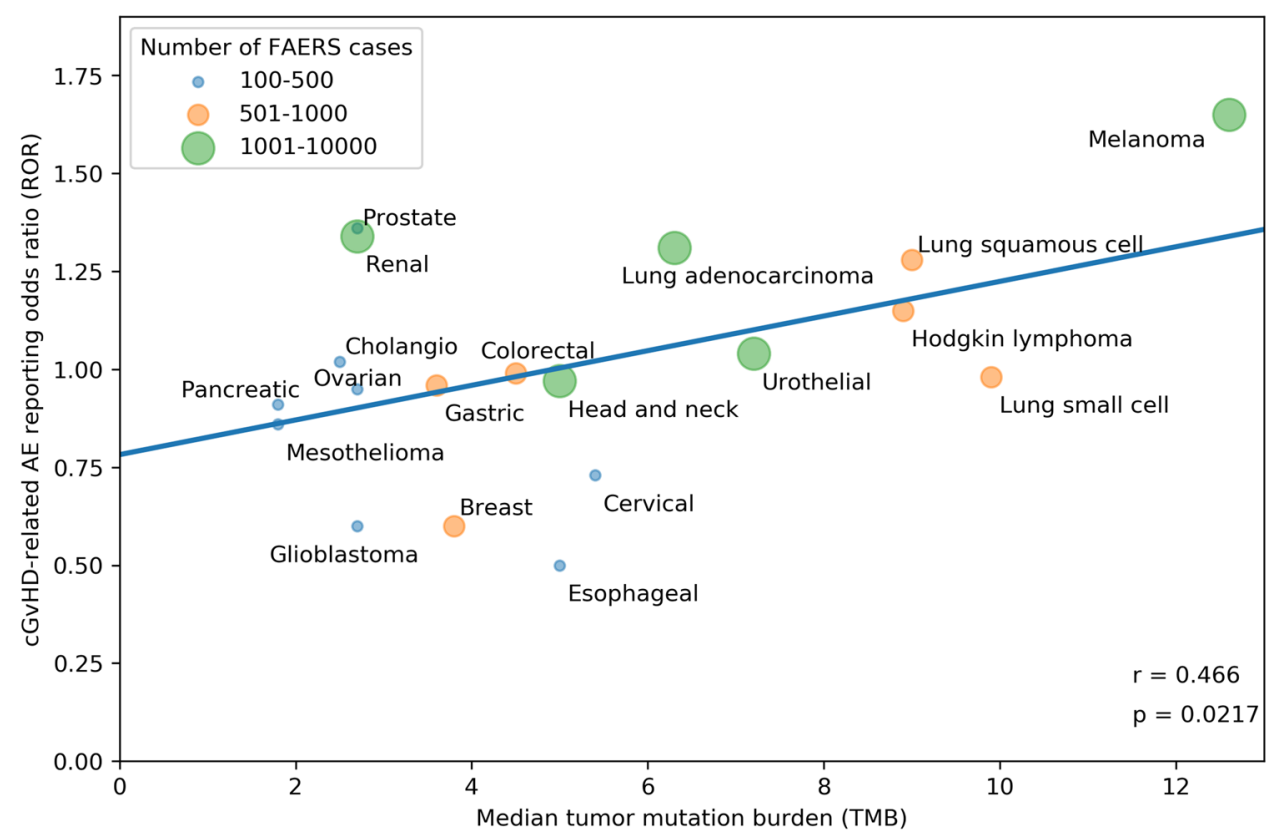


be highest in melanoma, lung squamous carcinoma, lung adenocarcinoma, bladder carcinoma, lung small cell carcinoma, esophageal carcinoma, colorectal carcinoma, cervix carcinoma, head and neck carcinoma. Melanomas have the highest mutational burdens (up to 100 mutations per megabase) as compared with other solid tumors.

The Kaplan-Meier curves for overall survival demonstrated a significant clinical benefit from CTLA-4 blockade in patients with melanoma with a neoepitope (nonsynonymous coding mutations) signature over those without the signature [14]. Consistent with this, Wang et al. [15], demonstrated in patients with advanced non-small cell lung carcinoma (NSCLC) that high TMB, estimated by circulating tumor DNA in blood (bTMB), was associated with superior progression-free survival and objective response rates to anti-PD-1 and anti-PD-L1 therapy compared to patients with low bTMB. Samstein et al. [16] also found that checkpoint inhibitors were more likely to halt tumor growth in patients with high TMB than in those with fewer mutations.

Our analysis indicates that cancers with a high TMB, such as melanoma, small cell and non-small cell lung cancers are associated with a higher irAE ROR during immune checkpoint inhibitor therapy. Since in our view neither anti-CTLA-4 nor anti-PD-1 antibodies are tumor specific [17], the development of irAEs depends upon the derangement of self-tolerance. Samstein et al. hypothesized that higher mutation load is associated with a higher number of tumor neoantigens that facilitate immune recognition and the development of an antitumor immune response [16]. In contrast, we proposed that this situation creates an autoGVHD, a concept not mentioned by Samstein et al. Specifically, as the number of TMB increases, tumor cells with newly expressed neoantigens are no longer recognized as "self" and transformed into targets for patient's own immune system cells. In other words, newly expressed neoantigens in malignant cells resulted in abrogation of the unresponsiveness/tolerance that existed between patient's immune system and cancer cells, thus allowing for development of autoGVHD with secondary therapeutic benefits, in analogy with GVM effects following allogeneic stem cell transplantation [2]. While a limited transformation is too weak in itself to provoke an effective $\mathrm{T}$ cell attack, the immune checkpoint blockade unleashes T cells against "altered-self" and tumors resulting in better overall survival [18]. This is consistent with the findings of Berner et al. [19], in non-small cell lung cancer. They demonstrated that $\mathrm{T}$ cells recognize and target shared tumor and skin antigens during checkpoint inhibitor therapy resulting in autoimmune-mediated skin toxicity and tumor regression. Not unexpectedly, the highest correlation $(r=0.678)$ was found between the TMB and ROR for $<$ rash $>$ among all AE terms in the FAERS database (Supplementary Table S3).
Acknowledgements Open access funding provided by Institute for Computer Science and Control. We thank David Bomze, MSc, and Lukas Flatz, MD, Institute of Immunobiology, Kantonsspital St Gallen, St Gallen, Switzerland, for helpful discussions.

Author contributions All authors had full access to all of the data in the study and take responsibility for the integrity of the data and the accuracy of the data analysis. Study concept and design: TB, CA, CK, RM. Acquisition, analysis, or interpretation of data: CK, TB, CA, SS. Drafting of the manuscript: TB, CA, RM, CK. Critical revision of the manuscript for important intellectual content: All authors. Computational and statistical data analysis: CK. Administrative, technical, or material support: CK, RM. Study supervision: SS, RM.

Funding CK was supported by the "2018-1.2.1-NKP-00008: Exploring the Mathematical Foundations of Artificial Intelligence" project supported by the Ministry of Human Capacities in Hungary. CA is supported by a Grant from the Canadian Institutes of Health Research (PS148588).

\section{Compliance with ethical standards}

Conflict of interest TB is a partner and CSO of PRET Therapeutics Ltd, developing the patented low-dose ICI combination therapy. All other authors have none to declare.

Ethical approval and ethical standards This study was exempt from ethical review because all analyzed datasets are deidentified and publicly available.

Informed consent Not applicable.

Open Access This article is licensed under a Creative Commons Attribution 4.0 International License, which permits use, sharing, adaptation, distribution and reproduction in any medium or format, as long as you give appropriate credit to the original author(s) and the source, provide a link to the Creative Commons licence, and indicate if changes were made. The images or other third party material in this article are included in the article's Creative Commons licence, unless indicated otherwise in a credit line to the material. If material is not included in the article's Creative Commons licence and your intended use is not permitted by statutory regulation or exceeds the permitted use, you will need to obtain permission directly from the copyright holder. To view a copy of this licence, visit http://creativecommons.org/licenses/by/4.0/.

\section{References}

1. Bomze D, Hasan Ali O, Bate A, Flatz L (2019) Association between immune-related adverse events during anti-PD-1 therapy and tumor mutational burden. JAMA Oncol. https://doi. org/10.1001/jamaoncol.2019.3221

2. Bakacs T, Moss RW, Kleef R, Szasz MA, Anderson CC (2019) Exploiting autoimmunity unleashed by low-dose immune checkpoint blockade to treat advanced cancer. Scand J Immunol. https ://doi.org/10.1111/sji.12821

3. Eggermont AMM, Kicinski M, Blank CU et al (2020) Association between immune-related adverse events and recurrence-free survival among patients with stage III melanoma randomized to receive pembrolizumab or placebo: a secondary analysis of a randomized clinical trial. JAMA Oncol. https://doi.org/10.1001/jamao ncol.2019.5570 
4. Callahan MK, Kluger H, Postow MA et al (2018) Nivolumab plus ipilimumab in patients with advanced melanoma: updated survival, response, and safety data in a phase I dose-escalation study. J Clin Oncol 36:391-398. https://doi.org/10.1200/ JCO.2017.72.2850

5. Xing P, Zhang F, Wang G et al (2019) Incidence rates of immunerelated adverse events and their correlation with response in advanced solid tumours treated with NIVO or NIVO + IPI: a systematic review and meta-analysis. J ImmunoTher Cancer 7:341. https://doi.org/10.1186/s40425-019-0779-6

6. Bate A, Evans SJ (2009) Quantitative signal detection using spontaneous ADR reporting. Pharmacoepidemiol Drug Saf 18:427436. https://doi.org/10.1002/pds.1742

7. Chalmers ZR, Connelly CF, Fabrizio D et al (2017) Analysis of 100,000 human cancer genomes reveals the landscape of tumor mutational burden. Genome Med 9:34. https://doi.org/10.1186/ s13073-017-0424-2

8. Liang WS, Vergilio JA, Salhia B et al (2019) Comprehensive genomic profiling of hodgkin lymphoma reveals recurrently mutated genes and increased mutation burden. Oncologist 24:219-228. https://doi.org/10.1634/theoncologist.2018-0058

9. Jagasia MH, Greinix HT, Arora M et al (2015) National institutes of health consensus development project on criteria for clinical trials in chronic graft-versus-host disease: I. The 2014 diagnosis and staging working group report. Biol Blood Marrow Transpl 21:389-401. https://doi.org/10.1016/j.bbmt.2014.12.001

10. Brahmer JR, Lacchetti C, Schneider BJ et al (2018) Management of immune-related adverse events in patients treated with immune checkpoint inhibitor therapy: American society of clinical oncology clinical practice guideline. J Clin Oncol 36:1714-1768. https ://doi.org/10.1200/JCO.2017.77.6385

11. Bakacs T, Mehrishi JN (2015) Anti-CTLA-4 therapy may have mechanisms similar to those occurring in inherited human CTLA4 haploinsufficiency. Immunobiology 220:624-625. https://doi. org/10.1016/j.imbio.2014.11.019

12. Slavin S, Moss RW, Bakacs T (2014) Control of minimal residual cancer by low dose ipilimumab activating autologous anti-tumor immunity. Pharmacol Res 79:9-12. https://doi.org/10.1016/j. phrs.2013

13. Alexandrov LB, Nik-Zainal S, Wedge DC et al (2013) Signatures of mutational processes in human cancer. Nature 500:415-421. https://doi.org/10.1038/nature12477

14. Snyder A, Makarov V, Merghoub T et al (2014) Genetic basis for clinical response to CTLA-4 blockade in melanoma. N Engl J Med 371:2189-2199. https://doi.org/10.1056/nejmoa1406498

15. Wang Z, Duan J, Cai S et al (2019) Assessment of blood tumor mutational burden as a potential biomarker for immunotherapy in patients with non-small cell lung cancer with use of a nextgeneration sequencing cancer gene panel. JAMA Oncol. https:// doi.org/10.1001/jamaoncol.2018.7098

16. Samstein RM, Lee CH, Shoushtari AN et al (2019) Tumor mutational load predicts survival after immunotherapy across multiple cancer types. Nat Genet 51:202-206. https://doi.org/10.1038/ s41588-018-0312-8

17. Bakacs T, Mehrishi JN, Moss RW (2012) Ipilimumab (Yervoy) and the TGN1412 catastrophe. Immunobiology. 217:583-589. https://doi.org/10.1016/j.imbio.2011.07.005

18. Thangavelu G, Murphy KM, Yagita H, Boon L, Anderson CC (2011) The role of co-inhibitory signals in spontaneous tolerance of weakly mismatched transplants. Immunobiology 216:918-924. https://doi.org/10.1016/j.imbio.2011.01.007

19. Berner F, Bomze D, Diem S et al (2019) Association of checkpoint inhibitor-induced toxic effects with shared cancer and tissue antigens in non-small cell lung cancer. JAMA Oncol. https://doi. org/10.1001/jamaoncol.2019.0402

Publisher's Note Springer Nature remains neutral with regard to jurisdictional claims in published maps and institutional affiliations. 